external causes were the most common COD among IDUs. Non AIDS mortality increased sixfold since 1997 (36-226), with particular increases in cardio/cerebrovascular disease, non-AIDS defining malignancies and external causes. Infectious diseases (mostly respiratory) was the most common underlying cause of non AIDS deaths for MSM and heterosexuals aged $15-59$ : (38\% and $36 \%$ respectively) and also high among IDUs (35\%). External causes (mostly due to overdose and suicide) was the most common COD among IDUs (36\%) and high among MSM (19\%). Cardio/cerebrovascular disease and non AIDS malignancies accounted for $16 \%$ and $11 \%$ of nonAIDS deaths (15-59 years) respectively. Three quarters $(76 \%)$ of all AIDS deaths and $47 \%$ of non AIDS deaths occurred within a year of diagnosis.

Conclusion Overall mortality rates in HIV positive persons have substantially declined in the HAART era but remain high compared to the general population. Clinical AIDS associated with late presentation continues to account for the majority of deaths. Our analyses also reveal that a disproportionate number of deaths are due to infectious diseases, overdose and suicide occur in this population, many of which may be preventable. Surveillance of nonAIDS causes of death is critical in the HAART era.

\section{P1-S3.05 ATTRIBUTABLE PROPORTION OF TUBAL FACTOR INFERTILITY CAUSED BY CHLAMYDIA: AN ESTIMATE BASED ON SEROLOGICAL EVIDENCE ADJUSTED FOR TEST RESOLUTION}

doi:10.1136/sextrans-2011-050108.138

M Price, A E Ades, J Macleod, P Horner. University of Bristol, Bristol, UK

Background Many published studies compare the prevalence of Chlamydia trachomatis (CT) antibody in women with Tubal Factor Infertility (TFI) and a control group. In principle these studies can be used to estimate the attributable fraction of TFI caused by CT, however it is necessary to account for the sensitivity and specificity of the antibody tests employed.

Methods We use sensitivity and specificity estimates from a discrepancy analysis by Morre (2002), and a study by Wills (2009) in which specificity of antibody test results was assessed in children, to derive estimates of the resolving power of three peptide-based assays and MIF; this is a reflection of the difference between sensitivity and false positive rates. Based on studies of antibody levels in different settings we adopt a model which assumes that antibody levels in women whose TFI is caused by CT are higher than levels in control women or those whose TFI has other causes. Applying this model to the data from Land (2003) we find strong support for the hypothesis of higher antibody levels in CT-related TFI, and for the estimates of test resolution from earlier studies. Using a range of assumptions about cumulative incidence of CT in the control group we were able to derive a range of estimates for the proportion of TFI cases caused by CT.

Results Our results suggest that the sensitivity of antibody tests in women whose TFI was caused by CT is higher than in women who have previously had CT but whose TFI was due to another cause and control women. Based on our estimates of the resolving power of the tests from the Morre (2002) and Wills (2009) studies we estimate the proportion of TFI episodes that are due to Chlamydia to be between $20 \%$ and $45 \%$. Conclusions By adjusting for the sensitivity and specificity of tests it is possible to derive a quantitative estimate of the causal rate of CT in TFI. Taken together with other findings our results suggests that detailed studies of antibody levels can be used to shed further light on the causal rate of CT.

\section{Epidemiology poster session 3: Burden of disease: PID \\ P1-S3.06 PREVALENCE AND DETERMINANTS OF NEISERIA GONORHOEA AND CHLAMYDIA INFECTIONS AMONG GYNAECOLOGICAL PATIENTS WITH PELVIC INFLAMMATORY DISEASE AT UNIVERSITY TEACHING HOSPITAL, LUSAKA}

doi:10.1136/sextrans-2011-050108.139

P Yassa. University Teaching Hospital, Lusaka, Zambia

Methodology This was a descriptive cross sectional study conducted on women presenting clinically with pelvic inflammatory disease to the outpatient department of obstetrics and gynaecology at University Teaching Hospital in Lusaka. Behavioural and demographic variable were collected through questionnaire. Endo-cervical smear was obtained and screened for gonorrhoea and Chlamydia using respective rapid test and gram stain for gonorrhoea.

Results 43 (37\%) of a total 116 respondents had gonorrhoea but no Chlamydia was isolated. 114 (98.3) had sexual partners. 101 had steady sexual partners, 19 had casual partners and 9 had anonymous sexual partners with 37 (36.6\%), 10 (52.6\%) and 6 (66.7\%) gonorrhoea isolation respectively. Some had multiple sexual partners. Gonorrhoea was isolated from 4 (28.6\%) of the 14 respondents who had one new sexual partner, and all who had two or more sexual partners had gonorrhoea isolated. Gonorrhoea isolation in relation to frequency of sexual intercourse per week was as follows: once 1/13 $(7.7 \%)$, twice $2 / 11(18.2 \%)$, thrice $3 / 11$ (27.3\%) and more than three times 32/65 (49.2). Gonorrhoea was also higher in those who had sex with casual or anonymous sexual partner under influence of alcohol $6 / 11(54.5 \%)$ or obtained anonymous sexual partner from Market, shopping canter $4 / 5(80 \%)$, street, bar, disco, or night club 7/9 (77.8\%). Gonorrhoea detection was as follows: 18/36 (50.0\%) for those with adnexial tenderness, 21/45 (46.7\%) with inflamed cervix, 37/92 (40.2\%) with lower abdominal tenderness and 32/87 (36.8\%) with normally appearing cervix.

Conclusion The prevalence of Neiseria gonorrhoea was 37\% detected. There was no Chlamydia isolated. Low socio-economic status and young age were the higher risk. The sexual risk behaviours associated were; the number of casual or anonymous sexual partners, and non-use of condoms. Lower abdominal pain and tenderness with cervical motion and adnexial tenderness were the major sign.

\section{P1-S3.07 RELATION BETWEEN FEMALE INFERTILITY AND SEXUALLY TRANSMITTED GENITAL INFECTIONS}

doi:10.1136/sextrans-2011-050108.140

C Almanza, M Ricardo, A González. Cira Garcia Clinic, Havana, Cuba

Background Sexually transmitted genital infections can bring about severe consequences, among them the leading cause of pelvic inflammatory desease (PID), which can lead to infertility. The objective of this study was to determine the relation between female infertility and sexually transmitted genital infections.

Methods An analytic study of cases and controls was carried out in Ramón González Coro Gyneco-obstetric Hospital, Cuba, 2009. The studied cases were 89 infertile women with tube obstruction who were assessed in infertility consultations, and the controls were carried out in 100 pregnant women who were about to give birth. Vaginal and endocervical secretion samples were taken. Genital micoplasma was present; it was determined through bacteriological culture techniques. Trichomonas vaginalis, Candida spp, Bacterial vaginosis, Neisseria gonorrhoeae, Chlamydia trachomatis, as well as the presence of other genital pathologicals; were investigated. The test 